\title{
A Mutation in the Cone-Specific pde6 Gene Causes Rapid Cone Photoreceptor Degeneration in Zebrafish
}

\author{
George Stearns, ${ }^{1}$ Meradelfa Evangelista, ${ }^{1}$ James M. Fadool, ${ }^{2}$ and Susan E. Brockerhoff ${ }^{1}$ \\ ${ }^{1}$ Department of Biochemistry, University of Washington School of Medicine, Seattle, Washington 98195, and 2Department of Biological Science, Florida \\ State University, Tallahassee, Florida 32306-4340
}

\begin{abstract}
Photoreceptor degeneration is a common cause of inherited blindness worldwide. We have identified a blind zebrafish mutant with rapid degeneration of cone photoreceptors caused by a mutation in the cone phosphodiesterase $c$ ( $p d e 6 c$ ) gene, a key regulatory component in cone phototransduction. Some rods also degenerate, primarily in areas with a low density of rods. Rod photoreceptors in areas of the retina that always have a high density of rods are protected from degeneration. Our findings demonstrate that, analogous to what happens to rod photoreceptors in the $r d 1$ mouse model, loss of cone phosphodiesterase leads to rapid degeneration of cone photoreceptors. Furthermore, we propose that cell density plays a key role in determining whether rod photoreceptors degenerate as a secondary consequence to cone degeneration. Our zebrafish mutant serves as a model for developing therapeutic treatments for photoreceptor degeneration in humans.
\end{abstract}

Key words: zebrafish; photoreceptor; phototransduction; retina; phosphodiesterase; degeneration

\section{Introduction}

Retinal photoreceptors are the primary cells in the eye responsible for detecting light. Without photoreceptors, we are unable to process visual stimuli, and our ability to function in the world is severely limited. Vertebrates contain two classes of photoreceptors, rods and cones. Rods are responsible for vision under dim illumination, whereas cones respond to bright light and give us color vision.

The enzymatic cascade responsible for light detection is known and is analogous in rod and cone photoreceptors. The seven-transmembrane GPCR (G-protein-coupled receptor) rhodopsin or cone opsin together with 11-cis-retinal, absorbs a photon of light. Activated opsin then interacts with transducin and converts it from inactive GDP-bound form, to an active GTPbound form. GTP-transducin stimulates a cGMP phosphodiesterase leading to the hydrolysis of cGMP and the closure of cation channels within the photoreceptor outer segment. This causes cell hyperpolarization and a decrease in vesicular glutamate release at the cell synapse (for review, see Chen, 2005).

Photoreceptor degeneration is one of the most common causes of inherited blindness. Dozens of genes responsible either for rod degeneration, cone degeneration, or the degeneration of both of these photoreceptor classes have been identified. For example, retinitis pigmentosa (RP), a disease characterized by the

Received July 10, 2007; revised 0ct. 26, 2007; accepted 0ct. 26, 2007.

This work was supported by National Institutes of Health (NIH) Grants EY015165 (G.S., S.E.B., M.E.) and EY017753 (J.M.F.). We thank Dr. Owen Lawrence for a critical reading of this manuscript and Dr. James B. Hurley for assistance with the IGOR program for analyzing ERG data. Finally, we thank Edward Parker and NIH Vision Core Grant EY01730 for assistance with electron microscopy.

Correspondence should be addressed to Susan E. Brockerhoff, Department of Biochemistry, Box 357350, University of Washington School of Medicine, Seattle, WA 98195. E-mail: sbrocker@u.washington.edu.

D01:10.1523/JNEUROSCI.3136-07.2007

Copyright $\odot 2007$ Society for Neuroscience ～0270-6474/07/2713866-09\$15.00/0 initial degeneration of rods followed by secondary degeneration of cones, can be caused by mutations in $\sim 35$ different genes (http://www.sph.uth.tmc.edu/Retnet/). Patients with RP are first night blind and then progressively lose all their vision.

The discovery of many disease genes causing photoreceptor degeneration has been facilitated by the specialized nature of rods and cones and the nonessential requirement of vision for survival; most of the genes involved in phototransduction are unique to photoreceptors. Unfortunately, the functional diversity in the types of defects leading to RP and other degenerative disorders has made identifying common molecular mechanisms underlying degeneration difficult (for review, see Hims et al., 2003; Hartong et al., 2006; Daiger et al., 2007). Even less understood in this devastating disease, are the molecular mechanisms that lead to the secondary death of photoreceptors not expressing the mutated gene. For example, why do cones die in RP when it is the rods that express the mutated gene?

In this report, we present a zebrafish cone photoreceptor degeneration mutant. Zebrafish are ideal for analyzing retinal degeneration. They develop rapidly, have strong easily measured visual responses, and can be easily manipulated using molecular and genetic methods. Through genetic screening and positional cloning, we identified a mutation in the cone-specific phosphodiesterase gene ( $p d e 6 c$ ). This mutation leads to the rapid degeneration of all cone photoreceptors soon after their formation. The rods also degenerate, but what is striking is that this degeneration occurs only in areas that were originally rich in cone photoreceptors and contained relatively few rod photoreceptors. Rod photoreceptors in areas of the zebrafish retina that always have a high density of rods appear protected from degeneration. Our findings demonstrate that, analogous to what happens in the $r d 1$ mouse model, loss of phosphodiesterase leads to rapid cone photoreceptor cell death. Furthermore, we propose that cell density plays a 
key role in determining whether photoreceptors degenerate as a secondary consequence to an initial burst of degeneration. Our zebrafish mutant serves as a model for developing therapies to treat photoreceptor degeneration in humans.

\section{Materials and Methods}

Zebrafish maintenance and mutant isolation. $\mathrm{AB}^{*}$ and WIK strain zebrafish were obtained from the University of Oregon and maintained as an inbred stock in the University of Washington Zebrafish Facility. Adult fish and larvae were maintained at $28.5^{\circ} \mathrm{C}$ in reverse-osmosis distilled water reconstituted for fish compatibility by addition of salts and vitamins (Westerfield, 1995) on a 10/14 h dark/light cycle.

The $p d e 6 c^{w 59}$ mutant was isolated in a three-generation screen of ethyl nitrosourea-mutagenized $\mathrm{AB}^{*}$ strain zebrafish using the optokinetic response (OKR) behavioral assay as described previously (Brockerhoff, 2006; Brockerhoff et al., 1998). Briefly, progeny from crosses between $F_{2}$ siblings were partially immobilized in $6 \%$ methylcellulose (Sigma, St. Louis, MO), and their eye movements were analyzed in response to rotating illuminated stripes. In crosses between $p d e 6 c^{w 59}$ heterozygotes, $25 \%$ of the larvae showed no eye movements in white light. The stripe width used for the screen was $20^{\circ}$. Under normal conditions, $p d e 6 c^{w 59}$ larvae do not survive to become adults. However, when we grew $p d e 6 c^{w 59}$ larvae with a $10 \times$ higher than normal concentration of paramecium, $50-100 \%$ of the mutant larvae would survive past 2 weeks postfertilization (pf). Between 14 and $21 \mathrm{dpf}, p d e 6 c^{w 59}$ mutants began to eat brine shrimp and then survived as well as wild-type (WT) larvae under the normal conditions of our fish facility. Fish that show a normal OKR $(\mathrm{OKR}+)$ include WT fish and fish heterozygous for the $p d e 6 c^{w 59}$ mutation. There were no obvious phenotypic differences in electrophysiology or histology between WT and heterozygous fish. For all experiments identifying and scoring polymorphisms, a hybrid strain between $\mathrm{AB}^{\star}$ and WIK was used. WIK is a WT zebrafish strain that is polymorphic with the $\mathrm{AB}^{\star}$ strain and is commonly used for mapping studies (Johnson and Zon, 1999). Because $p d e 6 c^{w 59}$ was generated in $\mathrm{AB}^{\star}$, this mutation segregated with $\mathrm{AB}^{\star}$ markers.

To visualize ON-bipolar cells, fish heterozygous for the $p d e 6 c^{w 59}$ allele were mated to fish carrying the nyx::MYFP transgene. This transgene directs expression of the membrane-targeted form of YFP (yellow fluorescent protein) in a subset of ON-bipolar cells (Schroeter et al., 2006).

Electroretinograms. The procedure for recording electroretinograms (ERGs) has been described previously (Van Epps et al., 2001). Zebrafish were dark-adapted for $1 \mathrm{~h}$ before placement in the recording chamber and were then left in complete darkness for an additional $10 \mathrm{~min}$ minimum after positioning.

Immunocytochemistry. Immunolabeling and fluorescence imaging were as previously described (Fadool, 2003). Briefly, eyes or whole larvae were fixed by immersion in $4 \%$ paraformaldehyde in $80 \%$ HBSS at $4^{\circ} \mathrm{C}$ for $4 \mathrm{~h}$ to overnight. Tissues were rinsed in buffer and cryoprotected in $30 \%$ sucrose and mounted in OCT medium (Miles Scientific, Elkhart, IN). Seven- to $10-\mu \mathrm{m}$-thick frozen sections were adhered to gelatincoated glass slides and postfixed in $2 \%$ paraformaldehyde. Immunofluorescent labeling using the mouse monoclonal antibodies that recognize specific cells in the zebrafish neural retina, in combination with mousespecific Alexa 543-conjugated secondary antibodies (Invitrogen, Carlsbad, CA) were performed essentially as described previously (Fadool, 2003). Nuclei were counter stained with 4',6-diamidino-2-phenylindole (DAPI) (Sigma). Tissues were viewed on the Zeiss (Oberkochen, Germany) inverted microscope, and images were captured and processed using the Zeiss Axiocam Digital Camera and Axiovision software. Confocal images were generated on a Zeiss LSM 510 equipped with a $40 \times$ water immersion objective [numerical aperture (NA), 1.2]. The following primary antibodies and dilutions were used: 1D1 (1:100 dilution), a monoclonal antibody that recognizes an epitope on rhodopsin; 4C12 (1:100 dilution), a monoclonal antibody that recognizes an unknown epitope on rods (J. Fadool and P. Linser, unpublished data); and Zpr-1 (1:20 dilution), a monoclonal antibody that recognizes red and green cones (Oregon Monoclonal Bank, Eugene, OR). For several ages, bipolar cells were immunolabeled with rabbit antibody to protein kinase $\mathrm{C} \alpha$ $(\mathrm{PKC} \alpha)$ (1:100 dilution; Santa Cruz Biotechnology, Santa Cruz, CA).
Histological analysis and cell counts. Light and transmission electron microscopy (TEM) on mutant and sibling OKR + larvae were done as described previously (Schmitt and Dowling, 1999). For TEM, 60-70 nm sections from two OKR + and two $p d e 6 c^{w 59}$ larvae were analyzed.

To quantify the number of rods or ON bipolar cells in the outer (ONL) or the inner nuclear layer (INL), respectively, retinal sections were examined from individual eyes from two to five mutant or age-matched $\mathrm{OKR}+$ fish per time point. Bipolar cells were counted in retinal sections from 8-9 dpf, $16 \mathrm{dpf}$, and 12-week-old fish (no differences in bipolar cell number between OKR + and mutant were observed at any of the time points examined). Rods were counted in 16 and 28 dpf larvae, and 6- and 12 -week-old fish (for rod cell numbers, see text). For adult retinas, two or three transverse sections taken through or adjacent to the optic nerve, and separated by a distance of no less than $70 \mathrm{~mm}$ were examined. Confocal stacks ( $5 \mathrm{~mm}$ thick) were captured from two regions of the dorsal retina approximately midway between the optic nerve and margin and a third region adjacent to the retinal margin using a $40 \times$ water immersion objective lens (NA, 1.2). For larvae, images of the entire eye or of the central retina were captured using a 20 or $40 \times$ objective lens, and a single section through or bordering the optic nerve was examined. Rod cell bodies were identified by immunolabeling with $4 \mathrm{C} 12$. Bipolar cells were either immunolabeled with antisera against PKC $\alpha$ or expressed the nyx::MYFP transgene (Schroeter et al., 2006). All samples were counterstained with DAPI. The number of cells in $50-100 \mathrm{~mm}$ regions was counted, and the data were expressed as the number of intact neurons per $100 \mu \mathrm{m}$ of linear length of the ONL. For statistical analysis, data were first tested for unequal variances and none was found. At each time point, differences between the average number of neurons in WT $(\mathrm{OKR}+)$ and mutant were tested using Student's $t$ test.

\section{Results}

\section{Mutant identification}

We use a behavioral screening strategy that identifies recessive mutations in $\mathrm{F}_{3}$ generation larvae. These larvae are derived from ENU ( $N$-ethyl- $N$-nitrosourea)-treated great grandfather fish. In our behavioral assay, we analyze the larvae at $5 \mathrm{dpf}$ for abnormal eye movements in response to rotating illuminated stimuli. This screening strategy identifies genes essential for cone visual pathways because zebrafish larvae rely exclusively on their cone photoreceptors for visual function (Brockerhoff et al., 1998).

Our first observation of the mutant larvae, at $5 \mathrm{dpf}$, was that they had no eye movements in response to the rotating illuminated stimuli. Otherwise, the mutant appeared completely normal in morphology (Fig. 1A,B). The external eye size appeared normal and mutant larvae developed a swim bladder, an indirect measure of fish health. The mutation is recessive and one-quarter of the larvae from a mating cross between two heterozygous fish are blind at $5 \mathrm{dpf}$.

\section{Mutant retinal histology and gene identification}

After identifying apparently blind fish with normal external morphology, we proceeded to analyze retinal histology in WT versus mutant fish. In WT zebrafish at $4 \mathrm{dpf}$, cone photoreceptors are already distributed throughout the retina in a precise mosaic (Raymond et al., 1995). Cones in the central retina are most mature and cones in the periphery are the least mature. In contrast, at $4 \mathrm{dpf}$, rod photoreceptors are less densely distributed throughout the retina and are initially concentrated in the ventral region, in an area referred to as the ventral patch (Raymond et al., 1995; Fadool, 2003).

By $4 \mathrm{dpf}$, we noticed that cones had already degenerated (Fig. $1 D$ ) in the mutant. The loss of cones is so extensive that in the place of cones there are holes or gaps (white areas) in the outer retina where the cones should reside (Fig. $1 D$ ). The degeneration we observed in the mutant at day 4 was in the central part of the 
retina. The ventrally located prominent rods appeared intact. Furthermore, by 7 $\mathrm{dpf}$, cells with rod-like morphology were distributed throughout the retina (Fig. $1 E)$. Interestingly, the rod outer segments appeared slightly aggregated at this age. Also, contact between the retinal pigment epithelium (RPE) and photoreceptors, was reestablished by $7 \mathrm{dpf}$. We hypothesize that the absence of the intervening cones causes neighboring rods to appear aggregated. These histological observations led us to conclude that the mutation initially leads to degeneration of cones and not rods.

After establishing that the mutant had an apparently cone-specific degeneration phenotype, we proceeded to map and clone the mutated gene. Through a positional/candidate gene approach, we identified $p d e 6 c$ as the gene mutated in our blind zebrafish (Fig. $2 \mathrm{~A}$ ). We first mapped the mutation to linkage group 12 using 500 mutant larvae as our mapping panel. pde6c is positioned in the SANGER (http://www. ensembl.org/Danio_rerio/index.html) zebrafish genomic sequence near this mutation. We considered $p d e 6 c$ a reasonable candidate gene based on the histological phenotype. Because the mutant cone photoreceptors degenerate, obtaining significant mutant mRNA from cone photoreceptors was challenging. Therefore, we amplified genomic DNA, together with flanking intron splice donor and acceptor sites, to identify the mutation. We found a single nucleotide change (A to $G$ ) between WT and mutant genomic DNA (Fig. 2A) that disrupts a splice acceptor site leading to a deletion of two nucleotides and a corresponding frame shift in the cDNA for pde6c (allele designation $p d e 6 c^{w 59}$ ). We predict that the stop codon at amino acid position 506 in the mutant reading frame leads either to nonsense-mediated decay of the mRNA or a truncated Pde6c protein, which is degraded in developing cones.

In situ hybridization with antisense probe on WT larvae confirmed the cone-specific expression of pde6c (Fig. $2 B-E$ ). At $53 \mathrm{~h}$, we initially saw pde6c message only in the pineal (Fig. 2C). At 72 hpf, mRNA was detected in the eye and in some larvae was confined to the ventral patch or was extending in the anterior, nasal direction (Fig. 2D). The staining then rapidly progressed throughout the ONL and evenly covered this layer in all larvae by day 4 (Fig. $2 E$ ). The initial progression and even distribution of staining by day 4 is characteristic of genes expressed specifically in cone photoreceptors (Raymond et al., 1995; Brockerhoff et al., 2003).

Once we had identified the mutation, we were able to genotype fish before when they could be identified by alteration of visual behaviors. We analyzed genotyped fish early in development by time-lapse imaging (data not shown) and also histology (Fig. 1C). Using these methods, we established that the cones begin degenerating at $\sim 80 \mathrm{hpf}$. We also labeled young genotyped
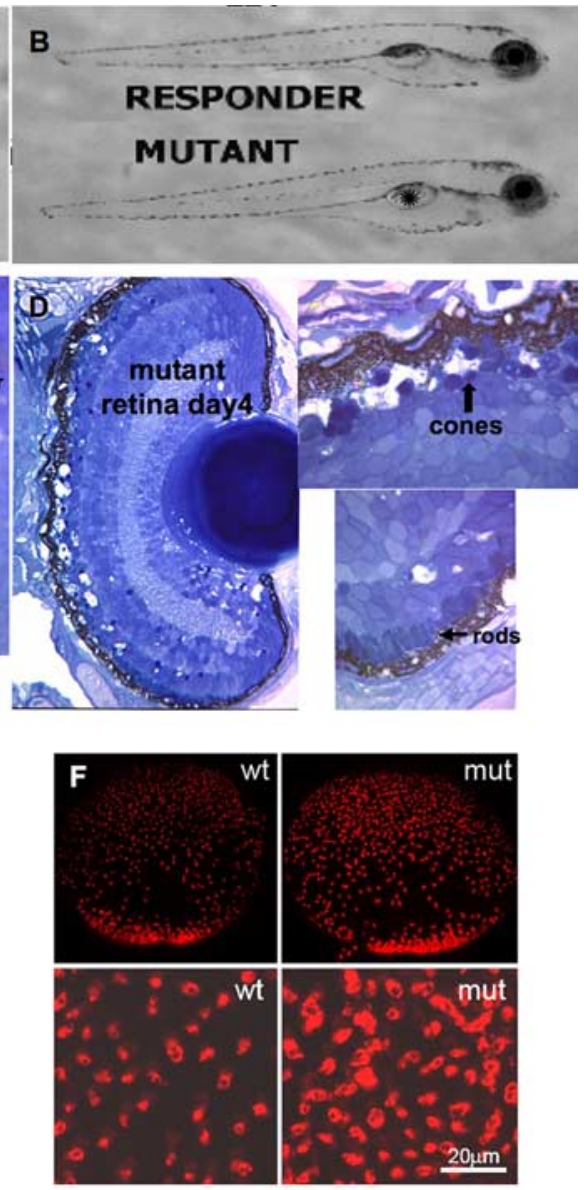

Figure 1. External morphology and retinal phenotype of PDE6c $-/-$ through $7 \mathrm{dpf}$. Dorsal $(\boldsymbol{A})$ and lateral $(\boldsymbol{B})$ views of $p d e 6 c^{w 59}$ and sibling nonblind fish. Notice the normal eye size and development of a swim bladder (asterisk in $\boldsymbol{B}$ ) in the mutant. The mutation is recessive and one-quarter of the larvae from a mating cross between two heterozygous fish are blind at $5 \mathrm{dpf}$ 作 panel labeled "rods" points to the elongated rod outer segments in the ventral patch of the retina. $\boldsymbol{E}$, At $7 \mathrm{dpf}$ in WT retinas, cone retis was collected at the level of photoreceptor outer segments. The overall distribution of rods across the retina appears similar in mutant and WT eyes. Rod outer segments are pronounced in the mutant. The antibody used (4c12) labels an unknown epitope specific to rod photoreceptors. Please see text for more details.

larvae with an antibody that specifically labels rod photoreceptors. As suggested by the histology, the immunocytochemistry on genotyped larvae indicated that rod photoreceptors are intact and, furthermore, that their distribution throughout the retina appeared normal (Fig. $1 F$ ), although the width of the outer segments appeared broader than in the OKR + siblings.

This type of progression of cone photoreceptor degeneration most closely resembles severe cone dystrophy, Leber congenital amaurosis, or an early-onset macular degeneration. It is analogous to rapid rod degeneration caused by loss of function (recessive) mutations in the rod-specific pde6 $\beta$ (Bowes et al., 1990; Ionita and Pittler, 2007).

\section{ERGs}

To assess the function of rods in the $p d e 6 c^{w 59}$ mutant, we recorded ERGs. The two initial components of an ERG are the $a$-wave and the $b$-wave. The $a$-wave records the electrical activity from stimulated photoreceptors. The $b$-wave records the activity 

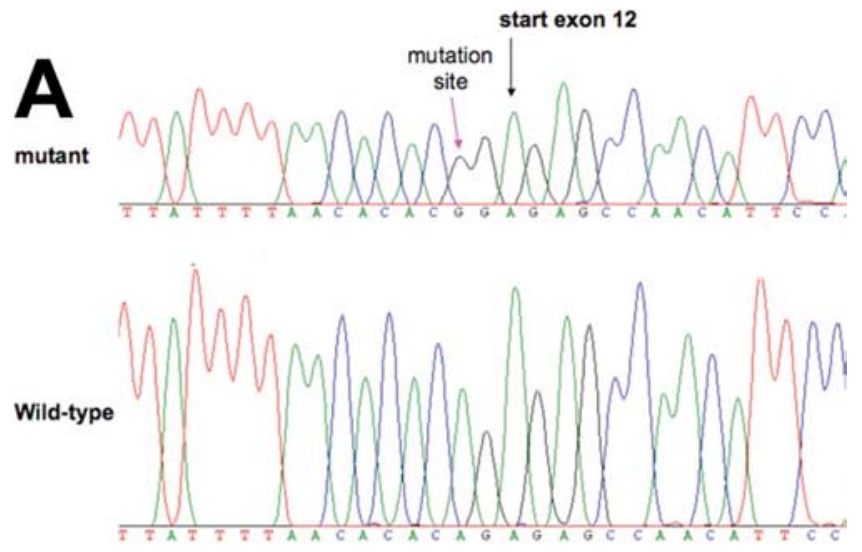

PDE6 partial sequence of intron and exon 12

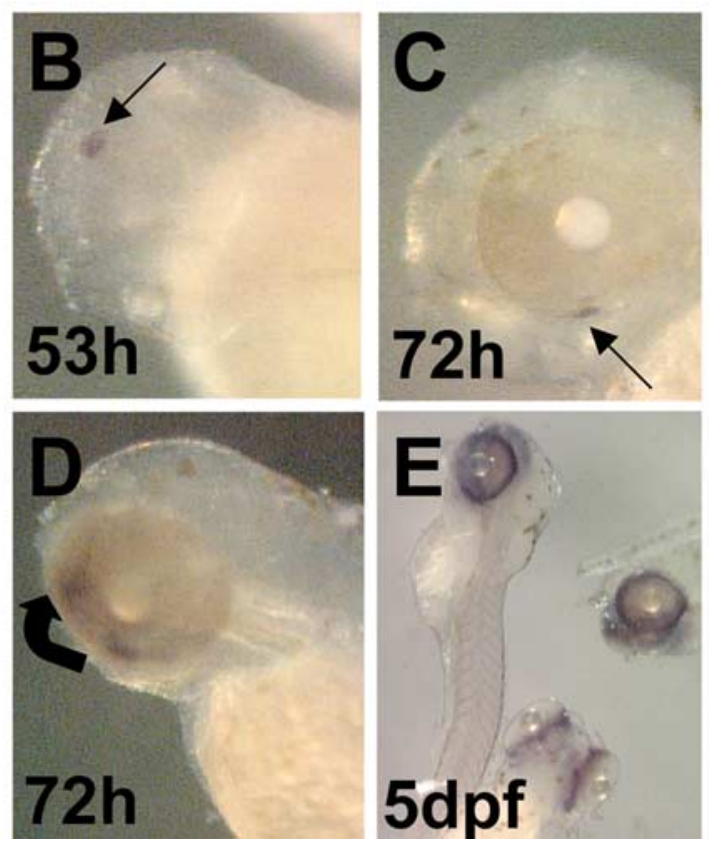

Figure 2. Sequence comparison between $p d e 6 c^{\mathrm{W} 59}$ fish and WT fish and cone-specific expression of $p d e 6 c . A$, The mutation is in a splice acceptor site between exon 11 and exon 12 . The red arrow points to the mutation $(A$ to $G$ ) and the black arrow marks the beginning of exon 12 . Mutant sequence is on top, and WT sequence is on the bottom. $\boldsymbol{B}-\boldsymbol{E}$ show in situ hybridization on WT zebrafish larvae using pde6c antisense RNA. B, At $53 \mathrm{hpf}$, staining was only detected in the pineal (arrow). C, D, At $72 \mathrm{hpf}$, staining in the ventral patch (arrow) and ventral anterior portion (leftward arrow) of the eye was detected. $\boldsymbol{E}$, Robust symmetric staining across the ONL was evident at $5 \mathrm{dpf}$.

from neurons postsynaptic to the photoreceptors (Dowling, 1987; Pugh and Lamb, 1993).

In zebrafish, the rod photoreceptors mature more slowly than the cones. Vision and ERG responses in WT $5 \mathrm{dpf}$ zebrafish larvae are determined by cones. In the absence of functional cones, the larval ERG response is absent (Brockerhoff et al., 2003). Thus, as expected, the all cone ERG of the $5 \mathrm{dpf}$ zebrafish larva was absent in the $p d e 6 c^{w 59}$ mutant (data not shown).

In wild-type zebrafish, the rod component of the ERG can be separated from the cone component at $\sim 2-3$ weeks postfertilization (Branchek, 1984; Bilotta et al., 2001). We hypothesized, given the pronounced rod outer segments in the $p d e 6 c^{w 59}$ fish at 4 and $7 \mathrm{dpf}$ (Fig. $1 E, F)$, that we might detect a rod ERG even earlier in the $p d e 6 c^{w 59}$ mutant than in WT larvae. However, to date,
ERGs done through $40 \mathrm{dpf}$ in the mutant show only a small $a$-wave component of the rod ERG (Fig. 3) (data not shown).

\section{Rod photoreceptor dystrophy in the absence of neighboring photoreceptors}

One possible explanation for the lack of a robust rod ERG is that the rods degenerate slowly as a secondary consequence to the rapid cone degeneration. To evaluate this hypothesis, we examined the retina from mutants at additional time points up through 6 weeks of age using histological methods (Figs. 4, 5).

Because the mutant $p d e 6 c^{w 59}$ larvae are blind, they are unable to actively forage for food and typically die from starvation at $\sim 10$ dpf. To grow larvae to older ages, we concentrated paramecia $\sim 10$-fold through low-speed centrifugation. At these high concentrations, $p d e 6 c^{w 59}$ larvae consumed enough paramecia to survive and grow. Once the $p d e 6 c^{w 59}$ larvae were large enough to eat brine shrimp, by $\sim 3$ weeks $p f$, the mutant larvae grew as large as sibling nonblind fish. This enabled analysis of retinal histology in young adult fish.

As shown in Figure 1, $E$ and $F$, at days 7 and 4, respectively, rod photoreceptors appeared abundant in the mutant retina with greater density in the ventral retina and more scattered labeling dorsally and centrally. However, when we analyzed mutant retinas $24-48 \mathrm{~h}$ later (at 8-9 dpf), we found that rod photoreceptors appeared somewhat reduced in number compared with $\mathrm{OKR}+$ and more strikingly rod outer segments appeared dystrophic (Fig. $4 B, D$ ). In fact, the rods in the central retina were so unhealthy that we could not easily quantify them because of a fragmented appearance. This reduction in rod number and abnormal rod morphology appeared most pronounced in the central retina.

We also found that bipolar cells in the mutant showed an abnormal morphology at this age in response to the massive cone degeneration (Fig. $4 E-H$ ). For example, in the INL of the OKR+ retina, the bipolar cell perikaria are normally aligned in a dense row, one to two cells thick. In contrast, many of the perikaria in the mutant larvae were displaced, residing either to the inner most region of the inner plexiform layer (IPL) or adjacent to the outer plexiform layer (OPL) (Fig. $4 E, F$ ). Furthermore, in the pde $6 c^{w 59}$ mutant retina, we observed misrouting of bipolar cell neurites beyond the IPL and into the ganglion cell layer (Fig. $4 F$, arrow), as well as gaps in the dendritic field of the OPL. Finally, in tangential confocal images, we noticed a dramatic reduction in the number and arrangement of dendritic processes in the OPL in the absence of the cones (Fig. 4G,H). However, bipolar cell number was the same in mutant and nonmutant retinas $(\mathrm{OKR}+, 67 \pm$ 4.1; pde $6 c^{w 59}, 68 \pm 5.2$ ) and terminal deoxynucleotidyl transferase-mediated biotinylated UTP nick end labelingpositive bipolar cells were not observed (data not shown). Alterations in morphology of second-order neurons and remodeling of dendritic processes are common features of photoreceptor cell dystrophies in humans and model organisms (Jones et al., 2003; Marc et al., 2003).

To verify the apparent secondary loss of rods in the central retina, we examined fish at $\sim 6$ weeks of age. Again, we found a noticeable loss of rods in the central retina where the majority of cones originally resided $\left(\mathrm{OKR}+, 59 \pm 4 ; p d e 6 c^{w 59}, 46 \pm 6\right)$. In the central retina, at most, we detected a single layer of photoreceptor nuclei between the pigmented epithelium and the OPL (Fig. $5 \mathrm{~A}$, red line). Furthermore, in many areas throughout the central retina, the OPL was completely absent and horizontal cells contacted photoreceptor nuclei (Fig. 5C,D, arrows). At 6 weeks, rod photoreceptors in the ventral region and also in the dorsal fourth of the retina were still abundant and we could identify intact rod 
synapses (Fig. $5 B$ ) as well as many rod nuclei (Fig. $5 A$, arrow). The loss of rods in the central region of the retina together with the dramatic loss of bipolar cell dendritic processes explains the absence of a rod ERG at 6 weeks of age.

The gradual degeneration of rods in the central retina in the $p d e 6 c^{w 59}$ larvae was very striking to us because it suggested additional secondary factors were influencing rod survival. We considered two possibilities: (1) that photoreceptor cell density was critical for determining cell survival (because rods in the central retina were primarily dying) or (2) that rods required neighboring cones for their survival. To distinguish between these possibilities, we were able to take advantage of a feature unique to zebrafish, that new photoreceptors are generated in the fish retina throughout life. Many teleosts and amphibians continue to grow throughout their life, and the increase in body mass is matched by an increase in the size of the eye and the area of the retina (Johns and Fernald, 1981; Fernald, 1990; Marcus et al., 1999; Otteson and Hitchcock, 2003). The increase in retinal area is attributable in part to the annular addition of new neurons, including photoreceptors, at the retinal margin by a population of mitotic progenitor cells. At the retinal margin, approximately equal numbers of rods and cones are generated. Another source of rod photoreceptors are proliferating cells within the INL and ONL (Raymond and Rivlin, 1987; Julian et al., 1998; Otteson et al., 2001). We predicted that, if photoreceptor cell density were a critical factor controlling rod survival, as the rod cell density at the retinal margin (and possibly in the center) increased sufficiently, the newly generated rods would continue to populate the retina. However, if the second scenario was correct and the absence of cones somehow poisoned rod viability, we would never see an abundance of rods in the central retina, although rods and cones would still be born in the periphery.

To test these ideas, we waited for mutant fish to reach 3 months of age to allow time for retinal outgrowth and then examined mutant and OKR + retinal histology. At 3 months, the rod photoreceptors appeared healthy throughout the retina $\left(\mathrm{OKR}+\right.$ central rods, $62.8 \pm 9.6$; pde6 $c^{w 59}$ central rods, $64.8 \pm$ 17.4) with elongated outer segments, several rows of cell nuclei (Fig. $6 B, D$ ) in the ONL, and apparently normal rod spherules (Fig. 6E, $F$, arrows). Therefore, in regions initially with a high density of rods, the ventral patch in the larvae (Fig. $1 D)$ and that generated by the retinal margin in the adult (Fig. $6 B, D$ ), the rods did not degenerate. Eventually, rods populated the entire outer retina, including the center, suggesting that rods generated by the rod progenitor cells also survive. These results are consistent with rods requiring neighboring photoreceptors for their survival and suggest that cell density influences photoreceptor survival.

We analyzed 3 month mutant retinas for cones as well. As expected, the cones continue to degenerate soon after formation. We observed greater numbers of newly generated cones at the retinal periphery (Fig. 6C), although their morphology was abnormal, with aberrant outer segments. In the more mature central retina, the cones were sparse (Fig. 6A), and large stretches of retina were completely devoid of cone photoreceptors (Fig. $6 \mathrm{~A}$, arrow). Preliminary 5-bromo-2'-deoxyuridine (BrdU) uptake experiments also suggest that the loss of cones is associated with a regenerative response previously observed after cytotoxic assault, light damage, or mechanical injury of the teleost retina (data not shown) (A. C. Morris, T. L. Scholz, C. E. Brockerhoff, and J. M. Fadool, unpublished observations) (see Discussion).

\section{Timeline of rod dystrophy and regeneration}

Our data suggest cell density influences rod survival. To determine the timeline of loss and regeneration of rods, we analyzed 16 and $28 \mathrm{dpf}$ OKR + and $p d e 6 c^{w 59}$ larvae as well. At these ages, we found the rods cells in the central retina so fragmented, the ONL to be incomplete, and the INL to be juxtaposed to the RPE, that it was impossible to obtain accurate counts of any remaining intact rods (data not shown). Together, our data indicate that rods die secondarily to cones in the central retina soon after the first week of development and that the subsequent rod regeneration requires months.

\section{Discussion}

This study reports a newly identified zebrafish mutant with a defect in the cone-specific pde6 gene. This mutant will be a useful model for understanding achromatopsia and photoreceptor degeneration in humans and discoveries in zebrafish will complement findings made in other model systems such as the mouse.

Heritable achromatopsia (color blindness) in humans affects 1/33,000 Americans (Alexander et al., 2007). It is caused by defects in cone phototransduction genes and leads to loss of cone function, rod monochromacy, loss of visual acuity, light sensitivity, and nystagmus. Cone degeneration may or may not be associated with the disease. In humans, mutations in the cyclic nucleotide gated channel $\alpha$ and $\beta$ subunits as well as cone-specific version of transducin are known to cause this disease (Kohl et al., 2000, 2002; Johnson et al., 2004). Furthermore, a very recent preliminary report indicates that mutations in cone-specific $p d e 6$ can be identified in families with achromatopsia (Wissinger et al., 2007).

This study has made several significant findings. The initial contribution is the identification and initial characterization of the $p d e 6 c^{w 59}$ mutation and phenotype. The $p d e 6 c^{w 59}$ mutation causes rapid cone-specific degeneration and sets the stage for dissecting the biochemistry that underlies degeneration caused by Pde6 deficiencies. For example, one of the oldest and most common models for studying retinal degenerations is the $r d 1$ mouse (Farber and Lolley, 1974). In this mouse, rod photoreceptors degenerate because of a mutation in the $\beta$-subunit of the cGMP phosphodiesterase, essential for phototransduction (Bowes et al., 1990; Ionita and Pittler, 2007). Although a key biochemical feature of the $r d 1$ mouse mutation that cyclic nucleotides are elevated (Farber and Lolley, 1974) has been known since the early 1970s, we are still not able to precisely describe what occurs on a molecular level to trigger degeneration. A correlation has been found in other retinal dystrophies between high 

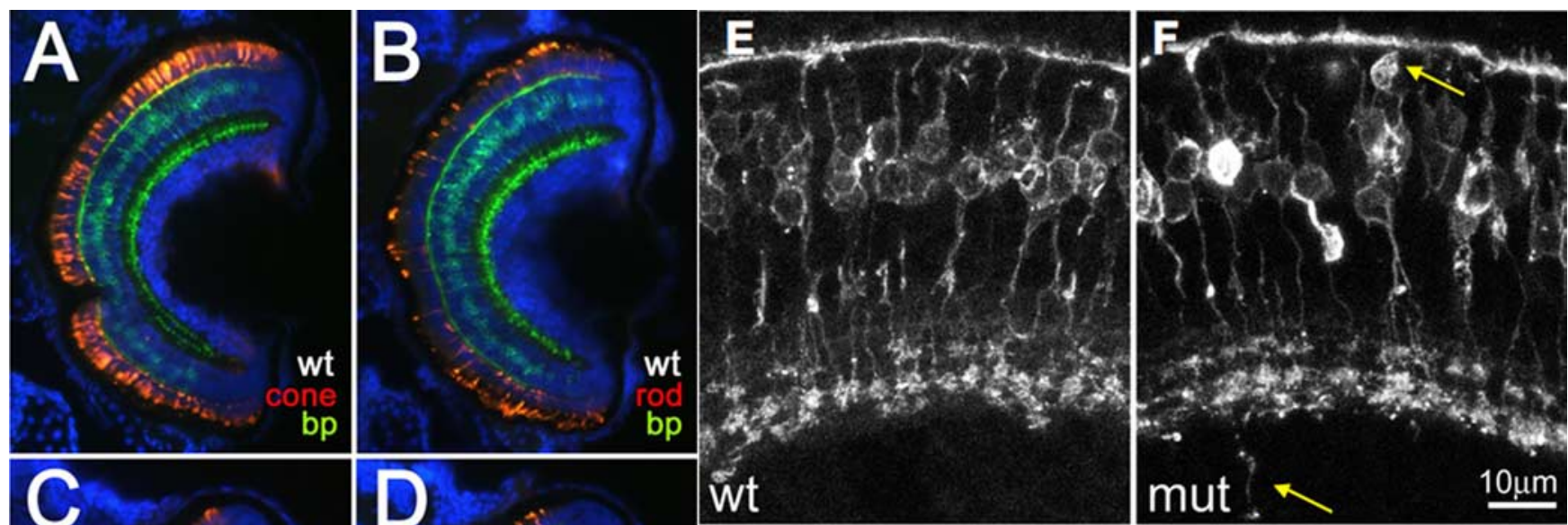
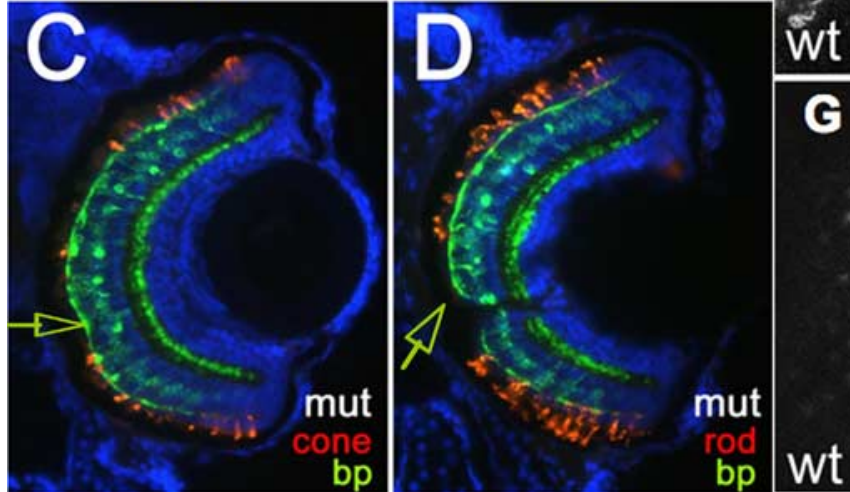

$\mathbf{G}$

Figure 4. Histology of WT and $p d e 6 c^{w 59}$ retinas at $8 \mathrm{dpf}$. $A$ and $($ show Fret 43 labeling for double cones. $B$ and $D$ show $4 c 12$ labeling for rods. Red, Antibody staining; blue, DAPI staining for nuclei green, nyx::MYFP for bipolar cells (bp) (Schroeter et al., 2006). In the WT retina, the cones $(\boldsymbol{A})$ are evenly distributed throughout the outer nuclear layer. The rods $(\boldsymbol{B})$ are less dense and their distribution is asymmetric but they also are found throughout the photoreceptor layer. In the $p d e 6{ }^{w 59}$ fish, cones $(\boldsymbol{C})$ and rods $(\boldsymbol{D})$ are localized to the retinal periphery. Note the absence of both rods and cones (arrows in $\boldsymbol{C}$ and $\boldsymbol{D}$ ) near the center of the retina, around the optic nerve. $\boldsymbol{E}-\boldsymbol{H}$ show high-magnification bipolar cell morphology in the WT and $p d e 6 c^{\text {w59 }}$. The arrows in $\boldsymbol{F}$ point to displaced bipolar nuclei and axonal processes in $p d e 6 c^{W 59}$. $G$ and $\boldsymbol{H}$ show a tangential section of bipolar dendrites at the level of the photoreceptor synapse. Note the dramatic reduction in number of dendritic processes and the lack of a regular pattern in the mutant compared with WT.
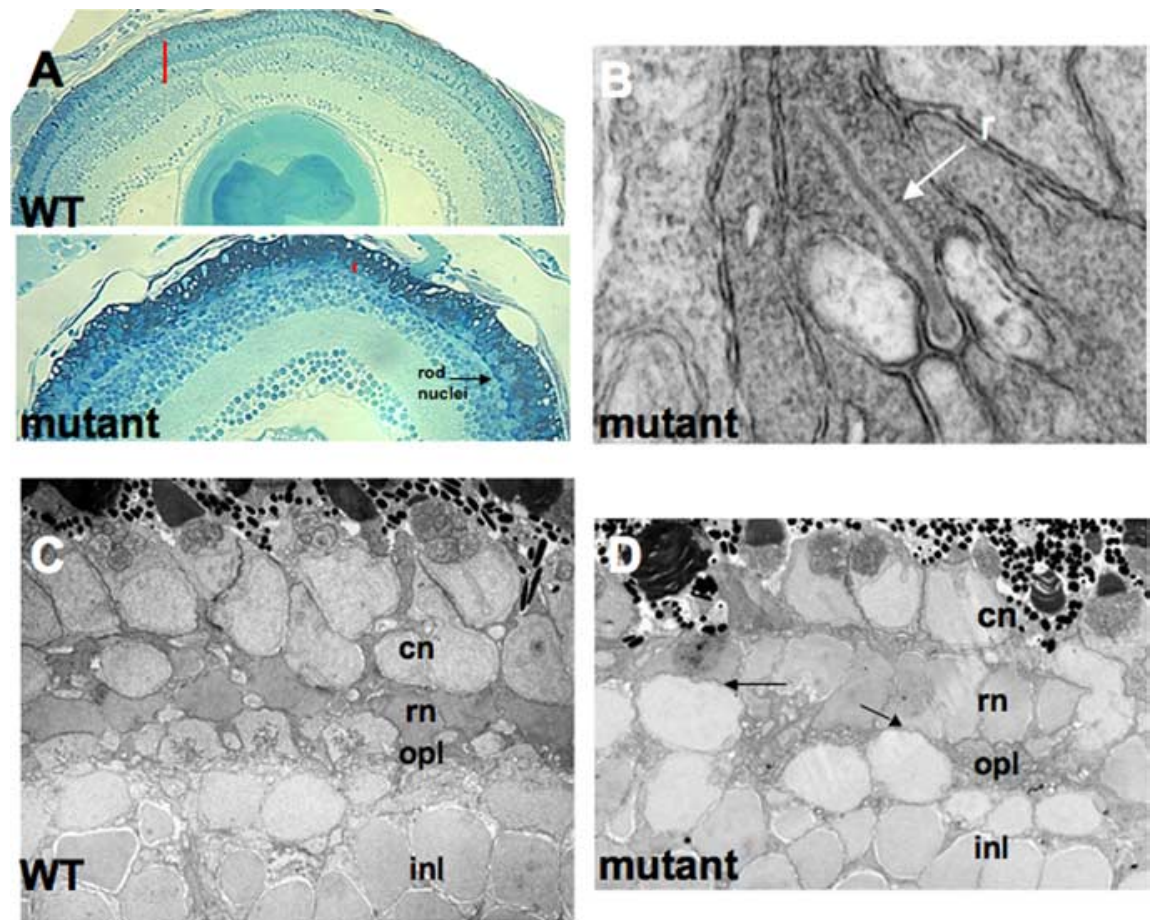

Figure 5. Histology of WT and $p d e 6 c^{w 59}$ retinas at 6 weeks. $A$ compares light micrographs of WT and mutant retinas. Note the proximity of the OPL to the pigmented epithelium (red line) in mutant compared with WT. The arrow in $A$ points to rod nuclei. $\boldsymbol{B}$ shows a morphologically normal rod spherule (r) from the $p d e 6 c^{w 59}$ retinal periphery. $\boldsymbol{C}$ and $\boldsymbol{D}$ compare the OPL of WT and $p d e 6 c^{w 59}$ central retinas. The arrows in $\boldsymbol{D}$ highlight regions in the mutant where the OPL is missing. cn, Cone nuclei; rn, rod nuclei. cyclic nucleotides and retinal degeneration (Olshevskaya et al., 2002). Furthermore, cGMP binds to plasma membrane cation channels in the outer segments of photoreceptors and maintains these channels in an open configuration. This stimulates an influx of calcium. The elevation of internal $\mathrm{Ca}^{2+}$ may be the apoptotic trigger but this idea has not been rigorously tested and data evaluating this possibility are inconsistent (Frasson et al., 1999; Bush et al., 2000; Pearce-Kelling et al., 2001; Pawlyk et al., 2002; Yamazaki et al., 2002; Takano et al., 2004; Vallazza-Deschamps et al., 2005).

The second major contribution made by this study, is the finding that cell density influenced photoreceptor death. What continues to remain a mystery in photoreceptor degenerative disorders is why photoreceptors that do not express the mutant gene die secondarily to photoreceptors expressing the mutation. We found that rod photoreceptors in regions initially of lower rod density and devoid of cones degenerated, whereas rods in regions containing higher numbers of rods (ventral patch) and the dorsal and ventral retinal margins survived. In the larval zebrafish, cones out- 
number rods by a factor of $8: 1$, but, in the adult, rods and cones are generated in approximately equal numbers (Fadool, 2003) (Fadool, unpublished observation). In teleost, including zebrafish, the increase in body mass throughout the life of the animal is matched by an increase in eye size and retinal area. Because all retinal neurons including photoreceptors continue to be generated from the retinal margin throughout the life of the fish, we could determine whether the absence of cones somehow caused rod degeneration. For example, if cones were essential for rod survival, we would never have rods populating the central retina in mutant fish, but this was not observed. Because at 3 months, we had a rod-dominated retina without cones across much of the retina, we hypothesize that cell number and not cell type influences the ability of rods to survive (for a schematic of our hypothesis, see Fig. 7). Previous studies indicate that, during retinal growth, $80 \%$ of the increase in retinal area occupied by photoreceptor cells is attributable to addition of new neurons from the retinal margin (Kock, 1982). We speculate that the majority of the new rods in the mutant are generated during outgrowth of the margin. Additionally, gradual filling in of rods from rod progenitors that reside in the central retina could occur in regions of the central retina bordering the areas of higher rod density followed by the progressive filling in toward the optic nerve. Our finding is consistent with what is known from the $r d 1$ mouse and human photoreceptor dystrophies. For example, in the analysis of patients with rhodopsin gene mutations, secondary cone disease correlated to the spatial and temporal onset of rod disease. Cone dysfunction by ERG and psychophysics was detectable with loss of $75 \%$ of the rod function (Cideciyan et al., 1998). In mice, rods are $\sim 20$ times more abundant than cones. Loss of rods in the $r d 1$ mouse and other mouse models of retinitis pigmentosa, leads secondarily to degeneration of cones (Carter-Dawson et al., 1978). Furthermore, in mouse mutants lacking cone cells or cone function, rods do not degenerate (Biel et al., 1999; Yang et al., 1999; Williams et al., 2005).

Recent studies in these mouse models of rod degeneration implicate oxidative damage as playing a major role in the secondary subsequent degeneration of cones. The hypothesis is that, after rods die, cones follow because of oxidative damage and recent experiments demonstrate that in the presence of antioxidants cone survival is improved (Komeima et al., 2006, 2007). This theory has been proposed because photoreceptors are known to consume large quantities of oxygen to generate the energy needed to maintain the dark current. Photoreceptors have one of the highest densities of mitochondria of any cell type in the body and the photoreceptor layer uses $50-100 \%$ of the oxygen consumed in the retina (Wangsa-Wirawan and Linsenmeier,
2003). It may be that a similar principle applies to rod degeneration in the $p d e 6 c^{w 59}$ fish and that an increase in cell density provides a "sink" for oxygen that minimizes cell damage attributable to oxidation.

Finally, the regenerative properties within the zebrafish retina provide the opportunity to determine factors contributing to cell fate determination and stem cell replacement therapies. For example, in preliminary experiments analyzing BrdU uptake in WT and $p d e 6 c^{w 59}$ fish, we found that proliferation within the INL was dramatically increased in the mutant (Morris, Scholz, Brockerhoff, and Fadool, unpublished observations). In a normal retina, proliferating cells within the INL and ONL generate rod cells (Raymond and Rivlin, 1987; Julian et al., 1998; Otteson et al., 2001). The abundance of rods at 3 months in the mutant retina compared with the mutant retina at 6 weeks would be consistent with an increase in rod progenitor proliferation. It is also known that the progenitors in the INL have the capability to become 


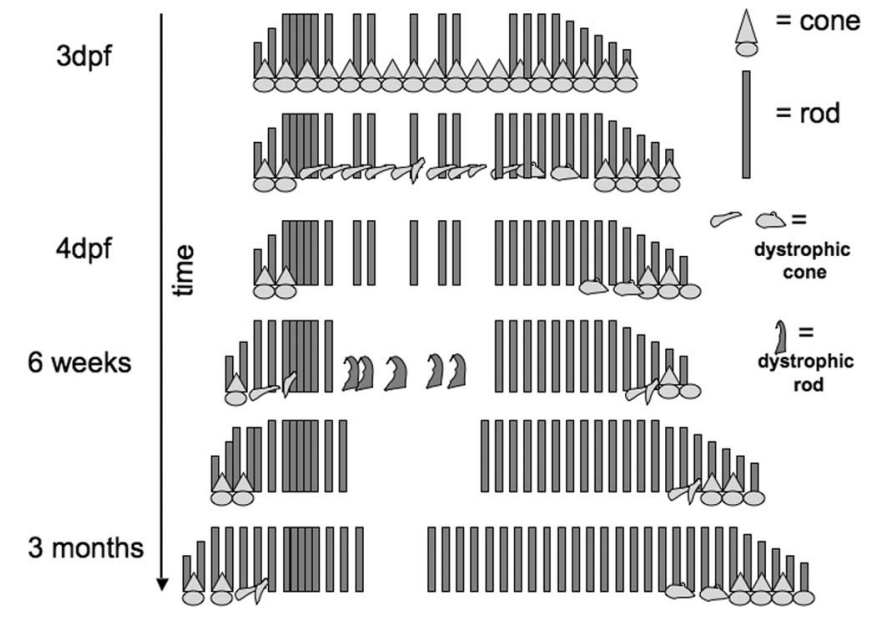

Figure 7. Schematic of cell loss and repopulation in the zebrafish pde6 ${ }^{w 59}$ mutant. At $3 \mathrm{dpf}$, cones are evenly distributed throughout the ONL and rods are less dense and asymmetrically distributed. Between 3 and $4 \mathrm{dpf}$, cones die. Subsequently, rods degenerate in the central retina. Cell growth at the retinal margins (and possibly also the within the INL and ONL) continues to generate new rods and cones. The cones continue to die because of the $p d e 6{ }^{\text {w5 }}{ }^{29}$ allele. The rod photoreceptors eventually repopulate the mutant retina.

other types of neurons in addition to rods in response to retinal injury (Fausett and Goldman, 2006; Bernardos et al., 2007; Fimbel et al., 2007). The identity of the proliferative cells identified in our preliminary study will require additional investigation. The availability of zebrafish mutations that cause rod degeneration (Morris et al., 2005), or all cone degeneration (this study), or selective cone type degeneration (Brockerhoff et al., 1997) as well as cell-selective ablation techniques (Davison et al., 2007; Pisharath et al., 2007), provide the opportunity to fine-tune our understanding of signals that induce proliferation and regulate cell fate determination.

In conclusion, we present a novel zebrafish mutation that serves as an animal model for a devastating form of blindness, achromatopsia. This model can be used to dissect the biochemistry underlying Pde-deficient photoreceptor degeneration as well as identify cues in cell fate determination and degeneration.

\section{References}

Alexander JJ, Umino Y, Everhart D, Chang B, Min SH, Li Q, Timmers AM, Hawes NL, Pang JJ, Barlow RB, Hauswirth WW (2007) Restoration of cone vision in a mouse model of achromatopsia. Nat Med 13:685-687.

Bernardos RL, Barthel LK, Meyers JR, Raymond PA (2007) Late-stage neuronal progenitors in the retina are radial Müller glia that function as retinal stem cells. J Neurosci 27:7028-7040.

Biel M, Seeliger M, Pfeifer A, Kohler K, Gerstner A, Ludwig A, Jaissle G, Fauser S, Zrenner E, Hofmann F (1999) Selective loss of cone function in mice lacking the cyclic nucleotidegated channel CNG3. Proc Natl Acad Sci USA 96:7553-7557.

Bilotta J, Saszik S, Sutherland SE (2001) Rod contributions to the electroretinogram of the dark-adapted developing zebrafish. Dev Dyn 222:564-570.

Bowes C, Li T, Danciger M, Baxter LC, Applebury ML, Farber DB (1990) Retinal degeneration in the rd mouse is caused by a defect in the beta subunit of rod cGMP-phosphodiesterase. Nature 347:677-680.

Branchek T (1984) The development of photoreceptors in the zebrafish, brachydanio rerio. II. Function. J Comp Neurol 224:116-122.

Brockerhoff SE (2006) Measuring the optokinetic response of zebrafish larvae. Nat Protoc 1:2448-2451.

Brockerhoff SE, Hurley JB, Niemi GA, Dowling JE (1997) A new form of inherited red-blindness identified in zebrafish. J Neurosci 20:1-8.

Brockerhoff SE, Dowling JE, Hurley JB (1998) Zebrafish retinal mutants. Vision Res 38:1335-1339.

Brockerhoff SE, Rieke F, Matthews HR, Taylor MR, Kennedy B, Ankoudinova
I, Niemi GA, Tucker CL, Xiao M, Cilluffo MC, Fain GL, Hurley JB (2003) Light stimulates a transducin-independent increase of cytoplasmic $\mathrm{Ca}^{2+}$ and suppression of current in cones from the zebrafish mutant nof. J Neurosci 23:470-480.

Bush RA, Kononen L, Machida S, Sieving PA (2000) The effect of calcium channel blocker diltiazem on photoreceptor degeneration in the rhodopsin Pro213His rat. Invest Ophthalmol Vis Sci 41:2697-2701.

Carter-Dawson LD, LaVail MM, Sidman RL (1978) Differential effect of the rd mutation on rods and cones in the mouse retina. Invest Ophthalmol Vis Sci 17:489-498.

Chen CK (2005) The vertebrate phototransduction cascade: amplification and termination mechanisms. Rev Physiol Biochem Pharmacol 154:101-121.

Cideciyan AV, Hood DC, Huang Y, Banin E, Li ZY, Stone EM, Milam AH, Jacobson SG (1998) Disease sequence from mutant rhodopsin allele to rod and cone photoreceptor degeneration in man. Proc Natl Acad Sci USA 95:7103-7108.

Daiger SP, Bowne SJ, Sullivan LS (2007) Perspective on genes and mutations causing retinitis pigmentosa. Arch Ophthalmol 125:151-158.

Davison JM, Akitake CM, Goll MG, Rhee JM, Gosse N, Baier H, Halpern ME, Leach SD, Parsons MJ (2007) Transactivation from Gal4-VP16 transgenic insertions for tissue-specific cell labeling and ablation in zebrafish. Dev Biol 304:811-824.

Dowling JE (1987) The retina: an approachable part of the brain. Cambridge, MA: Belknap, Harvard UP.

Fadool JM (2003) Development of a rod photoreceptor mosaic revealed in transgenic zebrafish. Dev Biol 258:277-290.

Farber DB, Lolley RN (1974) Cyclic guanosine monophosphate: elevation in degenerating photoreceptor cells of the $\mathrm{C} 3 \mathrm{H}$ mouse retina. Science 186:449-451.

Fausett BV, Goldman D (2006) A role for $\alpha 1$ tubulin-expressing Müller glia in regeneration of the injured zebrafish retina. J Neurosci 26:6303-6313.

Fernald RD (1990) Teleost vision: seeing while growing. J Exp Zool Suppl 5:167-180.

Fimbel SM, Montgomery JE, Burket CT, Hyde DR (2007) Regeneration of inner retinal neurons after intravitreal injection of ouabain in zebrafish. J Neurosci 27:1712-1724.

Frasson M, Sahel JA, Fabre M, Simonutti M, Dreyfus H, Picaud S (1999) Retinitis pigmentosa: rod photoreceptor rescue by a calcium-channel blocker in the rd mouse. Nat Med 5:1183-1187.

Hartong DT, Berson EL, Dryja TP (2006) Retinitis pigmentosa. Lancet 368:1795-1809.

Hims MM, Diager SP, Inglehearn CF (2003) Retinitis pigmentosa: genes, proteins and prospects. Dev Ophthalmol 37:109-125.

Ionita MA, Pittler SJ (2007) Focus on molecules: rod cGMP phosphodiesterase type 6. Exp Eye Res 84:1-2.

Johns PR, Fernald RD (1981) Genesis of rods in teleost fish retina. Nature 293:141-142.

Johnson S, Michaelides M, Aligianis IA, Ainsworth JR, Mollon JD, Maher ER, Moore AT, Hunt DM (2004) Achromatopsia caused by novel mutations in both CNGA3 and CNGB3. J Med Genet 41:e20.

Johnson SL, Zon LI (1999) Genetic backgrounds and some standard stocks and strains used in zebrafish developmental biology and genetics. Methods Cell Biol 60:357-359.

Jones BW, Watt CB, Frederick JM, Baehr W, Chen CK, Levine EM, Milam AH, Lavail MM, Marc RE (2003) Retinal remodeling triggered by photoreceptor degenerations. J Comp Neurol 464:1-16.

Julian D, Ennis K, Korenbrot JI (1998) Birth and fate of proliferative cells in the inner nuclear layer of the mature fish retina. J Comp Neurol 394:271-282.

Kock JH (1982) Neuronal addition and retinal expansion during growth of the crucian carp eye. J Comp Neurol 209:264-274.

Kohl S, Baumann B, Broghammer M, Jagle H, Sieving P, Kellner U, Spegal R, Anastasi M, Zrenner E, Sharpe LT, Wissinger B (2000) Mutations in the CNGB3 gene encoding the beta-subunit of the cone photoreceptor cGMP-gated channel are responsible for achromatopsia (ACHM3) linked to chromosome 8q21. Hum Mol Genet 9:2107-2116.

Kohl S, Baumann B, Rosenberg T, Kellner U, Lorenz B, Vadala M, Jacobson SG, Wissinger B (2002) Mutations in the cone photoreceptor G-protein alpha-subunit gene GNAT2 in patients with achromatopsia. Am J Hum Genet 71:422-425.

Komeima K, Rogers BS, Lu L, Campochiaro PA (2006) Antioxidants reduce 
cone cell death in a model of retinitis pigmentosa. Proc Natl Acad Sci USA 103:11300-11305.

Komeima K, Rogers BS, Campochiaro PA (2007) Antioxidants slow photoreceptor cell death in mouse models of retinitis pigmentosa. J Cell Physiol 213:809-815.

Marc RE, Jones BW, Watt CB, Strettoi E (2003) Neural remodeling in retinal degeneration. Prog Retin Eye Res 22:607-655.

Marcus RC, Delaney CL, Easter Jr SS (1999) Neurogenesis in the visual system of embryonic and adult zebrafish (Danio rerio). off. Vis Neurosci 16:417-424.

Morris AC, Schroeter EH, Bilotta J, Wong RO, Fadool JM (2005) Cone survival despite rod degeneration in XOPS-mCFP transgenic zebrafish. Invest Ophthalmol Vis Sci 46:4762-4771.

Olshevskaya EV, Ennilov AN, Dizhoor AM (2002) Factors that affect regulation of cGMP synthesis in vertebrate photoreceptors and their genetic link to human retinal degeneration. Mol Cell Biochem 230:139-147.

Otteson DC, Hitchcock PF (2003) Stem cells in the teleost retina: persistent neurogenesis and injury-induced regeneration. Vision Res 43:927-936.

Otteson DC, D'Costa AR, Hitchcock PF (2001) Putative stem cells and the lineage of rod photoreceptors in the mature retina of the goldfish. Dev Biol 232:62-76.

Pawlyk BS, Li T, Scimeca MS, Sandberg MA, Berson EL (2002) Absence of photoreceptor rescue with D-cis-diltiazem in the rd mouse. Invest Ophthalmol Vis Sci 43:1912-1915.

Pearce-Kelling SE, Aleman TS, Nickle A, Laties AM, Aguirre GD, Jacobson SG, Acland GM (2001) Calcium channel blocker D-cis-diltiazem does not slow retinal degeneration in the PDE6B mutant rcd 1 canine model of retinitis pigmentosa. Mol Vis 7:42-47.

Pisharath H, Rhee JM, Swanson MA, Leach SD, Parsons MJ (2007) Targeted ablation of beta cells in the embryonic zebrafish pancreas using E. coli nitroreductase. Mech Dev 124:218-229.

Pugh Jr EN, Lamb TD (1993) Amplification and kinetics of the activation steps in phototransduction. Biochim Biophys Acta 1141:111-149.

Raymond PA, Rivlin PK (1987) Germinal cells in the goldfish retina that produce rod photoreceptors. Dev Biol 122:120-138.

Raymond PA, Barthel LK, Curran GA (1995) Developmental patterning of rod and cone photoreceptors in embryonic zebrafish. J Comp Neurol 359:537-550.

Schmitt EA, Dowling JE (1999) Early retinal development in the zebrafish, Danio rerio: light and electron microscopic analyses. J Comp Neurol 404:515-536.

Schroeter EH, Wong RO, Gregg RG (2006) In vivo development of retinal ON-bipolar cell axonal terminals visualized in nyx::MYFP transgenic zebrafish. Vis Neurosci 23:833-843.

Takano Y, Ohguro H, Dezawa M, Ishikawa H, Yamazaki H, Ohguro I, Mamiya K, Metoki T, Ishikawa F, Nakazawa M (2004) Study of drug effects of calcium channel blockers on retinal degeneration of rd mouse. Biochem Biophys Res Commun 313:1015-1022.

Vallazza-Deschamps G, Cia D, Gong J, Jellali A, Duboc A, Forster V, Sahel JA, Tessier LH, Picaud S (2005) Excessive activation of cyclic nucleotidegated channels contributes to neuronal degeneration of photoreceptors. Eur J Neurosci 22:1013-1022.

Van Epps HA, Yim CM, Hurley JB, Brockerhoff SE (2001) Investigations of photoreceptor synaptic transmission and light adaptation in the zebrafish visual mutant nrc. Invest Ophthalmol Vis Sci 42:868-874.

Wangsa-Wirawan ND, Linsenmeier RA (2003) Retinal oxygen: fundamental and clinical aspects. Arch Ophthalmol 121:547-557.

Westerfield M (1995) The zebrafish book: a guide for the laboratory use of zebrafish (Brachydanio rerio). Eugene, OR: University of Oregon.

Williams GA, Daigle KA, Jacobs GH (2005) Rod and cone function in coneless mice. Vis Neurosci 22:807-816.

Wissinger B, Chang B, Dangel S, Hawes N, Hurd R, Jurklies B, Sener EC, Andreasson S, Kohl S, Heckenlively JR (2007) Cone phosphodiesterase defects in the murine cpfl1 mutant and human achromatopsia patients. J Invest Ophthalmol Vis Sci 48:4521.

Yamazaki H, Ohguro H, Maeda T, Maruyama I, Takano Y, Metoki T, Nakazawa M, Sawada H, Dezawa M (2002) Preservation of retinal morphology and functions in royal college surgeons rat by nilvadipine, a $\mathrm{Ca}^{2+}$ antagonist. Invest Ophthalmol Vis Sci 43:919-926.

Yang RB, Robinson SW, Xiong WH, Yau KW, Birch DG, Garbers DL (1999) Disruption of a retinal guanylyl cyclase gene leads to cone-specific dystrophy and paradoxical rod behavior. J Neurosci 19:5889-5897. 